\title{
PRESCRIPTION PATTERN OF ANTIMICROBIAL AGENTS AMONG DENTAL PRACTITIONERS IN A TERTIARY CARE CENTER IN NORTH INDIA
}

\author{
MOHAMMAD SUHAIB ${ }^{1 *}$, FARIDA AHMAD ${ }^{1}$, MOHAMMED AHMAD \\ ${ }^{1}$ Department of Pharmacology, Jawaharlal Nehru Medical College, Aligarh Muslim University, Aligarh - 202 002, Uttar Pradesh, \\ India. ${ }^{2}$ Department of Conservative Dentistry, Ziauddin Ahmad Dental College, Aligarh Muslim University, Aligarh - 202 002, \\ Uttar Pradesh, India. Email: 2011sohaib@gmail.com
}

Received: 09 December 2016, Revised and Accepted: 13 January 2017

ABSTRACT

Objective: The purpose of the study was to find out the pattern of antimicrobial prescription among dental practitioners in a tertiary care center in North India.

Methods: A total of 100 prescriptions of the patients attending the various dental outpatient departments of Ziauddin Ahmad Dental College, Aligarh Muslim University were collected and tabulated between January 2016 and June 2016. An observational study was done on the obtained prescriptions on various aspects like dental pathology for which antimicrobial were prescribed, antimicrobial agents used for management of acute and chronic conditions along with their dose and duration. Compliance of the patients during therapy and adverse effects associated with the regimen was also taken into consideration, and the final data were expressed as counts and percentages.

Results: Out of a total of 100 patients, 54 (54.0\%) were males while females were numbered as 46 (42.0\%) with maximum distribution of the patients between 31 and 40 years. On analysis, the most commonly presented dental condition for which antimicrobials were prescribed were acute/chronic periodontitis (48\%), acute/chronic gingivitis (32\%), caries (11\%), post root canal treatment (4\%), pericoronitis (3\%), oral trauma $(1 \%)$, and other condition (1\%). For the management of above conditions, antimicrobials were the most extensively used agents which were prescribed for only 3-5 days which may not be sufficient, followed by analgesics and antimicrobial mouthwashes. Among the antimicrobials used, most frequently used agents were amoxicillin (50\%) followed by ornidazole (21\%), ofloxacin (20\%), doxycycline (16\%), cefixime (9\%), and ciprofloxacin (3\%) used either alone or in combination for the management of acute and chronic conditions. Only two patients were advised for culture and sensitivity tests before antimicrobial therapy because of noncompliance of patients. The majority (74\%) of patients completed the prescribed regimen of antimicrobials while 7 patients reported adverse drug reactions (ADRs), among which most common were nausea and vomiting.

Conclusions: In our study, we found that the periodontal diseases were the most frequently reported conditions followed by acute/chronic gingivitis for which antimicrobials were prescribed while amoxicillin and ornidazole were the most commonly used antimicrobials for the management of various acute and chronic dental conditions, but were prescribed without culture and sensitivity in most cases. $7 \%$ patients reported ADRs. To conclude appropriate measures are to be promoted for rational prescribing and ADR reporting.

Keywords: Amoxicillin, Dental, Antimicrobials, infections, Prescription pattern.

(C) 2017 The Authors. Published by Innovare Academic Sciences Pvt Ltd. This is an open access article under the CC BY license (http://creativecommons. org/licenses/by/4. 0/) DOI: http://dx.doi.org/10.22159/ajpcr.2017.v10i4.16525

\section{INTRODUCTION}

Dental practitioners very often encounter odontogenic infections [1] in the form of periodontitis, gingivitis, pulpitis, abscess and post root canal treatment (RCT) and post extraction infections which makes the prescription of oral antimicrobials an important aspect in dental practice. Antimicrobials along with analgesics (including paracetamol and aceclofenac) and chlorhexidine mouthwashes $(0.1 \%)$ are most commonly prescriptions [2]. Antimicrobial therapy is usually indicated in oral conditions such as periodontal abscess, acute necrotizing ulcerative gingivitis, and pericoronitis [3]; however, they can be empirically started for the infections accompanied by fever, lymphadenopathy, and trismus [4,5]. However, injudicious and irrational use of antibiotics including selection of antimicrobial without prior culture and sensitivity tests, inadequate dosing, duration of treatment, proper follow-up is now major factor contributing to resistance among oral pathogens $[6,7]$ and thus decreasing the efficacy of antimicrobials. It also leads to adverse effects ranging from gastrointestinal upsets to life-threatening anaphylactic shock, emergence of resistant strains, and increased therapeutic cost $[8,9]$. Thus because of above reasons scrutiny of prescriptions showing antimicrobials choice, dosage, duration of therapy, and its efficacy are crucial for estimating rationality of their usage, evaluation of practices and modification in the pattern which may improve the standards of prescribing attitude in the region $[10,11]$.

\section{METHODS}

This was an observational study conducted at the Department of Pharmacology, JN Medical College, Aligarh Muslim University (AMU), Aligarh under which prescriptions of over 100 patients presenting to various outpatient departments of Ziauddin Ahmad Dental College, AMU, Aligarh were collected from January 2016 to June 2016 over a period of 6 months. Various aspects like dental conditions for which antimicrobial were prescribed, dose and duration of antimicrobial agents for the management of acute and chronic conditions were analyzed and tabulated. Compliance of the patients during therapy and adverse effects associated with the regimen was also taken into consideration, and the final data were expressed as counts and percentages. Finally, conclusions were figured out regarding the current practices and trends for the use of antimicrobial choices in different orodental infections and adverse effects and patient compliance related to them. 


\section{RESULTS}

In this study, a total of 115 prescriptions from dental practitioners were studied, out of which 100 were included in the study. The rest was excluded due to lack of patient compliance and discontinuation of antimicrobial therapy. The majority of patients visiting the dental practitioners were males (54\%) and were maximum in age group of 31-40 years followed by age group of 21-30 years (Table 1 and Chart 1 ).

The most common dental infections for which antimicrobials were prescribed were acute/chronic periodontitis (48\%), acute/chronic gingivitis (32\%), caries (11\%), post-RCT (4\%), pericoronitis (3\%), and oral trauma (1\%) (Table 2 and Chart 2).

The antimicrobials most frequently used for management of above-mentioned conditions were amoxicillin (50\%) followed by ornidazole (21\%), ofloxacin (20\%), doxycycline (16\%), cefixime $(9 \%)$, and ciprofloxacin $(3 \%)$ used either alone or in combination (Table 3 and Chart 3 ).

For acute infections amoxicillin was used in 17 (51.5\%) patients followed by cefixime and ornidazole which were advised in 5 (15.1\%) and 4 (12.1\%) patients, respectively (Chart 4).

However for management of chronic conditions most frequently advised agents were amoxicillin 33 (37.5\%), ornidazole 17 (19.3\%), ofloxacin 17 (19.3\%), and doxycycline 15 (17.0\%) (Chart 5).

Only two patients having chronic periodontitis with Type 2 diabetes mellitus were advised culture and sensitivity tests before antimicrobial therapy. Adverse drug reactions (ADRs) were reported by 4 patients, with nausea and vomiting being most commonly reported. Patient compliance was satisfactory (86.9\%), as 100 out of 115 patients completing the recommended course of antimicrobials.

\section{DISCUSSION}

The purpose of the study was to evaluate the antimicrobial prescribing practices among dental practitioners including the various dental pathologies for which antimicrobial agents were given and also the

Table 1: Distribution of patients according to age and gender

\begin{tabular}{llll}
\hline $\begin{array}{l}\text { Age group of } \\
\text { patients (years) }\end{array}$ & $\begin{array}{l}\text { Number of } \\
\text { patients (n) }\end{array}$ & Males (n) & Females (n) \\
\hline $0-10$ & 00 & 00 & 00 \\
$11-20$ & 11 & 04 & 07 \\
$21-30$ & 30 & 21 & 09 \\
$31-40$ & 31 & 11 & 20 \\
$41-50$ & 20 & 14 & 06 \\
$51-60$ & 07 & 03 & 04 \\
$61-70$ & 01 & 01 & 00 \\
$71-80$ & 00 & 00 & 00 \\
Total & 100 & 54 & 46 \\
\hline
\end{tabular}

Table 2: Frequency distribution of the conditions for which antimicrobial was prescribed

\begin{tabular}{llll}
\hline $\begin{array}{l}\text { Conditions for which } \\
\text { antimicrobials were advised }\end{array}$ & $\begin{array}{l}\text { Acute } \\
\text { cases }\end{array}$ & $\begin{array}{l}\text { Chronic } \\
\text { cases }\end{array}$ & Total \\
\hline Periodontitis & 05 & 43 & 48 \\
Gingivitis & 12 & 20 & 32 \\
Caries & 00 & 11 & 11 \\
Post-RCT & 04 & 00 & 04 \\
Pericoronitis & 03 & 00 & 03 \\
Oral trauma & 01 & 00 & 01 \\
Others & 01 & 00 & 01 \\
Total & 26 & 74 & 100 \\
\hline
\end{tabular}

RCT: Root canal treatment selection, dosage, duration of the antimicrobials advised to the patients. A total of 100 prescriptions were collected and assessed. On tabulating,

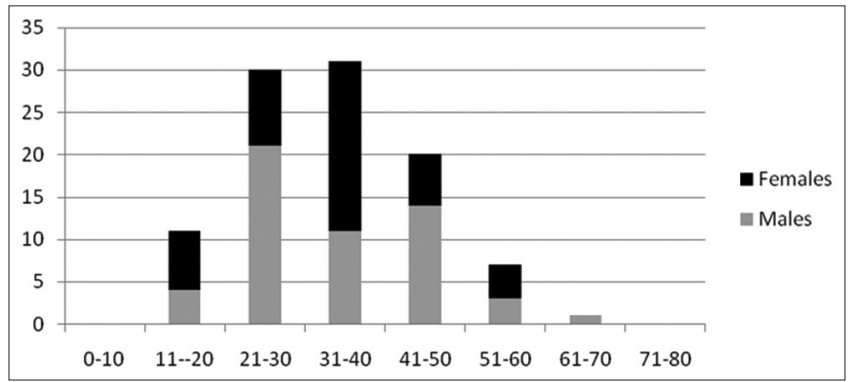

Chart 1: Distribution of patients according to age and gender

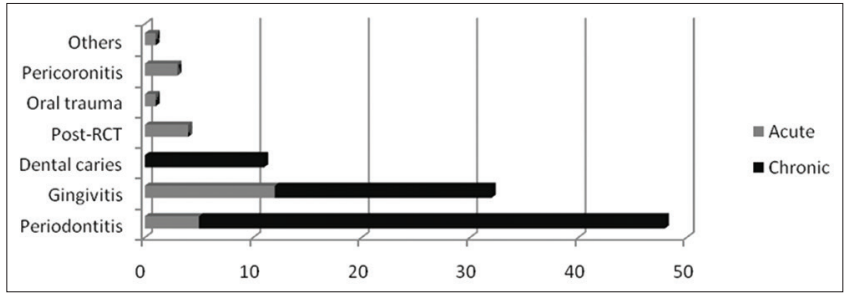

Chart 2: Frequency distribution of the conditions for which antimicrobial was prescribed

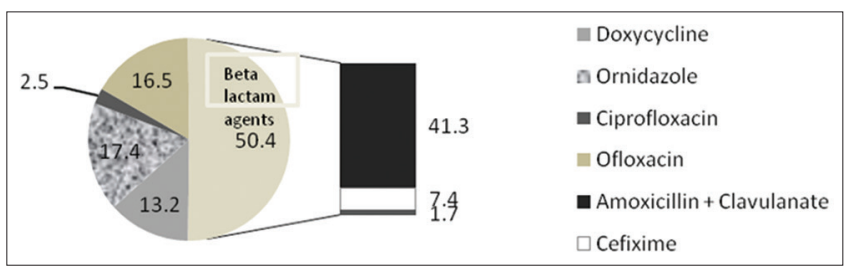

Chart 3: Frequency distribution of antimicrobials prescribed for dental conditions in percentage

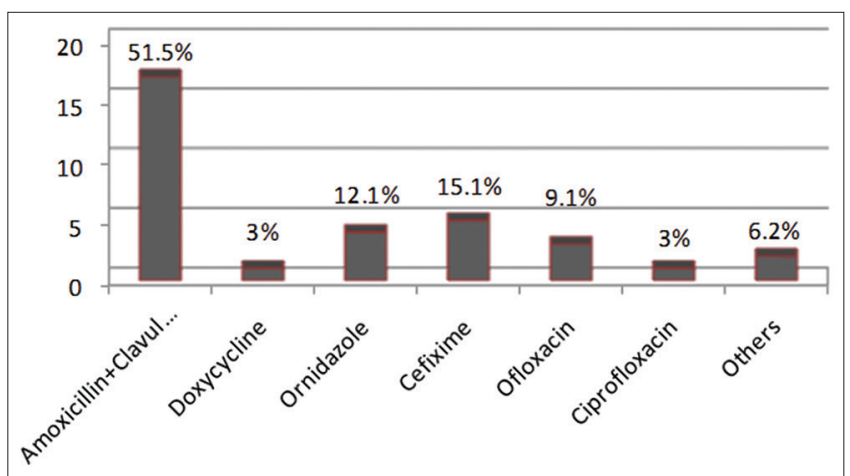

Chart 4: Pattern of antimicrobials prescribed in acute conditions

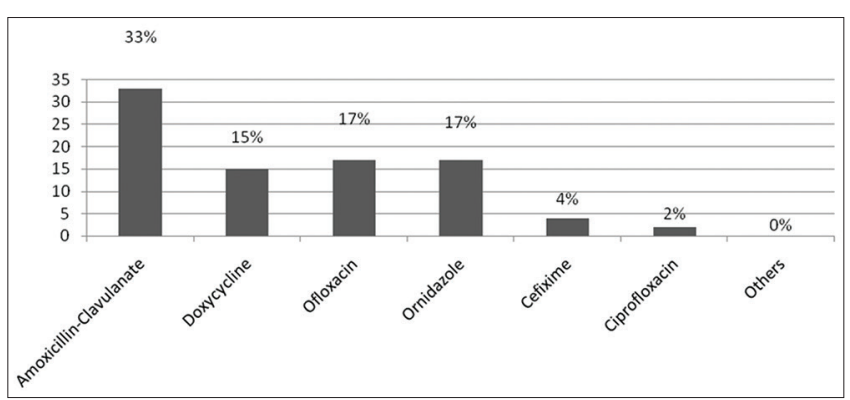

Chart 5: Antimicrobials prescription pattern in chronic condition 
the data from the prescriptions we found maximum patients (31\%) between age group 31 and 40 years with overall predominance of male cases (54\%) which may be correlated with the poor oral hygiene and tobacco chewing practice in the region. For evaluation of the antimicrobial prescribing pattern, reported infections were divided into acute and chronic conditions. Most common diagnosis for which antimicrobial agent was advised was periodontitis (48\%) of which $43(89.6 \%)$ cases were having chronic periodontitis while $5(10.4 \%)$ patients had acute periodontitis. It was followed by gingivitis (32\%) with $12(37.5 \%)$ acute gingivitis and $20(62.5 \%)$ chronic gingivitis patients. Other disease conditions reported were dental caries (11\%), post-RCT infections (4\%), pericoronitis (3\%), oral trauma cases (1\%), and others (1\%) which were case of maxillary swelling. In our study, we found that acute gingivitis 12 (46.1\%) was most common acute condition followed by acute periodontitis $5(19.2 \%)$ and post-RCT subjects 4 (15.3\%) while chronic periodontitis 43 (58.1\%), chronic gingivitis 20 (27.0\%), and dental caries $11(14.8 \%)$ were among most frequent chronic condition for which antibiotic was prescribed. Similar findings were also reported by a study in North India [12] which suggested soft tissue infection and chronic periodontitis as most common acute and chronic conditions treated with antimicrobials, respectively (Table 4).

Furthermore, in our study, we found that overall most frequently used antimicrobials was amoxicillin-clavulanate cotherapy 50 (41.3\%) followed by doxycycline 16 (13.2\%), ornidazole 21 (17.4\%), cefixime 9 (7.4\%), ofloxacin 20 (16.5\%), and ciprofloxacin $3(2.5 \%)$. Furthermore, we found that among the above agents amoxicillinclavulanate $(51.5 \%)$, cefixime $(15.1 \%)$ ornidazole $(12.1 \%)$ were most frequently used for acute cases while amoxicillin-clavulanate (37.5\%), ornidazole (19.3\%), ofloxacin (19.3\%), and doxycycline (17.0\%), were commonly prescribed for chronic conditions. Above findings were in accordance with the Antibiotics Prescribing Guidelines [13,14] which recommends amoxicillin or metronidazole as first choice agents for the management of acute cases while for metronidazole and doxycycline as first and second choice agents in chronic conditions. Other studies from
England and Iran also suggested amoxicillin as first choice agent in acute pathologies where it was prescribed $70.5 \%$ and $74.5 \%$, respectively, in those countries while beta-lactam antimicrobials (penicillins and cephalosporins 42\%), nitroimidazoles (24\%), fluoroquinolonesnitroimidazoles $(15 \%)$, and tetracyclines $(4 \%)$ were most commonly used for managing chronic cases in England [15-17]. Another reason for such an extensive use of amoxicillin may be its comparable clinical efficacy to other antibacterials, favorable dosage, and tolerability [18]. Although we found most of the prescriptions in accordance with the Antibiotic Prescribing Guidelines, the dose and duration of therapy of antimicrobials were inadequate in many prescriptions as shown in Table 3. Furthermore, we were disappointed to observe that only a few culture sensitivity tests were performed before antimicrobial therapy which may be due to lack of patients compliance and delay in management as culture reports are available only after $72 \mathrm{hrs}$. Furthermore, the prescription of probiotic supplements along with antimicrobials was only $4 \%$, thus increasing the risk of damage to intestinal commensal flora and associated diarrhea $[19,20]$.

\section{CONCLUSIONS}

Our findings suggested periodontal diseases as the most frequently reported conditions followed by acute/chronic gingivitis for which antimicrobials were prescribed in a dental college in North India. Amoxicillin-clavulanate followed by cefixime was the most commonly used antimicrobials for the management of acute conditions, while amoxicillin-clavulanate, ornidazole, and ofloxacin were the agents prescribed for chronic infections. However, the selection of antimicrobials was not confirmed with culture and sensitivity tests in most of the cases. 7\% patients reported ADRs of which nausea and diarrhea were most frequent. To conclude, considerable improvement is still required in creating professional awareness toward rational and test based antimicrobial prescription to control unnecessary adverse effects caused by the therapy and emergence of antimicrobial resistance.

Table 3: Details of dosage, frequency, and duration of prescribed antimicrobials

\begin{tabular}{|c|c|c|c|c|}
\hline Antimicrobials & Dose (mg) & Frequency (no of times a day) & Duration (Days) & Number of prescriptions \\
\hline \multirow[t]{4}{*}{ Amoxicillin-clavulanate } & 625 & Thrice & 5 & 26 \\
\hline & 125 & Twice & 3 & 10 \\
\hline & & & 5 & 08 \\
\hline & & & 3 & 06 \\
\hline \multirow{4}{*}{ Ofloxacin } & 400 & Twice & 5 & 04 \\
\hline & 200 & Twice & 3 & 09 \\
\hline & & & 5 & 04 \\
\hline & & & 3 & 03 \\
\hline \multirow[t]{2}{*}{ Ornidazole } & 500 & Twice & 5 & 08 \\
\hline & & & 3 & 13 \\
\hline Doxycycline & 100 & 2 tablets stat + twice daily for 14 days & 14 & 16 \\
\hline \multirow[t]{2}{*}{ Cefixime } & 200 & Twice & 5 & 02 \\
\hline & & & 3 & 07 \\
\hline Ciprofloxacin & 500 & Twice & 5 & 03 \\
\hline \multicolumn{5}{|l|}{ Others } \\
\hline Lincomycin & 500 & Twice & 5 & 01 \\
\hline Cefpodoxime & 200 & Twice & 3 & 01 \\
\hline
\end{tabular}

Table 4: Distribution of conditions for which antimicrobials were prescribed

\begin{tabular}{llll}
\hline Conditions for which antimicrobials were advised & Acute cases n (\%) & Chronic cases n (\%) & Total n (\%) \\
\hline Amoxicillin-clavulanic acid & $17(51.5)$ & $33(37.5)$ & $50(41.3)$ \\
Ornidazole & $4(12.1)$ & $17(19.3)$ & $21(17.4)$ \\
Ofloxacin & $3(9.1)$ & $17(19.3)$ & $20(16.5)$ \\
Doxycycline & $1(3.0)$ & $15(17.0)$ & $16(13.2)$ \\
Cefixime & $5(15.1)$ & $4(4.6)$ & $9(7.4)$ \\
Ciprofloxacin & $1(3.0)$ & $2(2.3)$ & $3(2.5)$ \\
Others & $2(6.2)$ & $0(0)$ & $2(1.7)$ \\
\hline
\end{tabular}




\section{ACKNOWLEDGMENTS}

Authors express our sincere thank to all the dental practitioners for their contributions.

\section{REFERENCES}

1. Dar-Odeh N, Ryalat S, Shayyab M, Abu-Hammad O. Analysis of clinical records of dental patients attending Jordan University Hospital: Documentation of drug prescriptions and local anesthetic injections. Ther Clin Risk Manag 2008;4(5):1111-7.

2. Lewis MA. Why we must reduce dental prescription of antibiotics: European Union Antibiotic Awareness Day. Br Dent J 2008;205(10):537-8

3. Salako NO, Rotimi VO, Adib SM, Al-Mutawa S. Pattern of antibiotic prescription in the management of oral diseases among dentists in Kuwait. J Dent 2004;32(7):503-9.

4. Swift JQ, Gulden WS. Antibiotic therapy - Managing odontogenic infections. Dent Clin North Am 2002;46(4):623-33, vii.

5. Bennadi D. Antimicrobial stewardship - An alarming call in dentistry. Int J Pharm Pharm Sci 2014;6(2):46-9.

6. Weber JT, Courvalin P. An emptying quiver: Antimicrobial drugs and resistance. Emerg Infect Dis 2005;11(6):791-3.

7. Standing Medical Advisory Committee. Sub-Group on Antimicrobial Resistance. The Path of Least Resistance. London: Stationery Office; 1998.

8. Craig WA, Uman SJ, Shaw WR, Ramgopal V, Eagan LL, Leopold ET. Hospital use of antimicrobial drugs. Survey at 19 hospitals and results of antimicrobial control program. Ann Intern Med 1978;89:793-5.

9. Dar-Odeh NS, Abu-Hammad OA, Al-Omiri MK, Khraisat AS, Shehabi AA. Antibiotic prescribing practices by dentists: A review.
Ther Clin Risk Manag 2010;6:301-6.

10. WHO. Studies in Drug Utilization. European Series No. 8. Copenhagen: WHO Regional Publications; 1979.

11. Al-Haroni M, Skaug N. Incidence of antibiotic prescribing in dental practice in Norway and its contribution to national consumption. J Antimicrob Chemother 2007;59(6):1161-6.

12. Gour PR, Kohli S, Advani U. Prescription pattern of antimicrobial agents by dental practitioners: A questionnaire based study. Int J Basic Clin Pharmacol 2013;2(3):311-4.

13. Drug Prescribing for Dentistry. Dental Clinical Guidance. $2^{\text {nd }}$ ed. August, 2011. Available from: http://www.sdcep.org.uk/index. aspx?o=2334. [Last accessed on 2013 Feb 15].

14. Antibiotic Prescribing Guidelines for Dentists. NHS Cambridge Shire and Peterborough Clinical Commissioning Group. Available from: http://www.extranet.cambridgeshire.nhs.uk/./Dental\%20information. [Last accessed on 2013 Feb 15].

15. Al-Haroni M, Skaug N. Knowledge of prescribing antimicrobials among Yemeni general dentists. Acta Odontol Scand 2006;64(5):274-80.

16. Palmer NA, Pealing R, Ireland RS, Martin MV. A study of therapeutic antibiotic prescribing in National Health Service general dental practice in England. Br Dent J 2000;188(10):554-8.

17. Vessal G, Khabiri A, Mirkhani H, Cookson BD, Askarian M. Study of antibiotic prescribing among dental practitioners in Shiraz, Islamic Republic of Iran. East Mediterr Health J 2011;17(10):763-9.

18. Kaur SP, Rao R, Nanda S. Amoxicillin: A broad spectrum antibiotic. Int J Pharm Pharm Sci 2011;3(3):30-7.

19. Marsh PD, Martin MV. Oral Microbiology. $4^{\text {th }}$ ed. Oxford: Wright; 1999. p. 210-1.

20. Lacey RW, Lord VL, Howson GL, Luxton DE, Trotter IS. Double-blind study to compare the selection of antibiotic resistance by amoxycillin or cephradine in the commensal flora. Lancet 1983;2:529-32. 